\title{
Lie derivatives and structure Jacobi operator on real hypersurfaces in complex projective spaces II
}

\author{
Juan de Dios Pérez and David Pérez-López
}

\begin{abstract}
Let $M$ be a real hypersurface in complex projective space. The almost contact metric structure on $M$ allows us to consider, for any nonnull real number $k$, the corresponding $k$-th generalized Tanaka-Webster connection on $M$ and, associated to it, a differential operator of first order of Lie type. Considering such a differential operator and Lie derivative we define, from the structure Jacobi operator $R_{\xi}$ on $M$ a tensor field of type $(1,2), R_{\xi_{T}}^{(k)}$. We obtain some classifications of real hypersurfaces for which $R_{\xi_{T}}^{(k)}$ is either symmetric or skew symmetric.
\end{abstract}

2000 Mathematics Subject Classification: 53C15, 53B25.

Keywords and phrases: $k$-th generalized Tanaka-Webster connection; complex projective space; real hypersurface; Lie derivative; structure Jacobi operator.

\section{Introduction.}

Consider a complex projective space $\mathbb{C} P^{m}, m \geq 2$, endowed with the complex structure $J$ and the Fubini-Study metric $g$ of constant holomorphic sectional curvature 4 . Let $M$ be a connected real hypersurface of $\mathbb{C} P^{m}$ without boundary, $\nabla$ its Levi-Civita connection on $M$ and $N$ a local unit normal vector field on $M$. Then $\xi=-J N$ is a tangent vector field to $M$ called the structure vector field on $M$ (or the Reeb vector field). The Kaehlerian structure $(J, g)$ of $\mathbb{C} P^{m}$ induces on $M$ an almost contact metric structure $(\phi, \xi, \eta, g)$, see [1], where $\phi$ is the tangent component of $J, \eta$ is an 1-form given by $\eta(X)=g(X, \xi)$ for any $X$ tangent to $M$ and $g$ is the metric induced on $M$.

Takagi, see [11], 12], [13], classified homogeneous real hypersurfaces in $\mathbb{C} P^{m}$ into 6 types. Kimura, [6], proved that these 6 types are the unique Hopf real hypersurfaces in $\mathbb{C} P^{m}$ with constant principal curvatures. A real hypersurface $M$ is called Hopf if the structure vector field is principal, that is, $A \xi=\alpha \xi$ for a certain function $\alpha$ on $M$, where $A$ denotes the shape operator of $M$ associated to $N$. We will also denote by $\mathbb{D}$ the maximal holomorphic distribution on $M$, given by all vector fields orthogonal to $\xi$. 
Among the real hypersurfaces appearing in Takagi's list we mention the following ones:

Type $\left(A_{1}\right)$ real hypersurfaces are geodesic hyperspheres of radius $r, 0<r<\frac{\pi}{2}$. They have 2 distinct constant principal curvatures, $2 \cot 2 r$ with eigenspace $\mathbb{R}[\xi]$ and $\cot r$ with eigenspace $\mathbb{D}$.

Type $\left(A_{2}\right)$ are tubes of radius $r, 0<r<\frac{\pi}{2}$, over totally geodesic complex projective spaces $\mathbb{C} P^{n}, 0<n<m-1$. They have 3 distinct constant principal curvatures, $2 \cot 2 r$ with eigenspace $\mathbb{R}[\xi]$, cot $r$ and $-\tan r$. The corresponding eigenspaces of $\cot r$ and $-\tan r$ are complementary and $\phi$-invariant distributions in $\mathbb{D}$.

From now on we will call type $(A)$ real hypersurfaces to both of either type $\left(A_{1}\right)$ or type $\left(A_{2}\right)$. The other real hypersurfaces appearing in Takagi's list have either 3 or 5 distinct constant principal curvatures.

Tanaka, [14], and, independtly Webster, [16], studied the canonical affine connection defined on a non-degenerate, pseudo-Hermitian CR-manifold, known as the TanakaWebster connection. Tanno, [15], generalized this connection for contact metric manifolds.

Cho, using the almost contact metric structure on a real hypersurface $M$ of $\mathbb{C} P^{m}$, generalized Tanno's connection, defining, for any non-zero real number $k$, the $k$-th generalized Tanaka-Webster connection $\hat{\nabla}^{(k)}$, see [2], [4], by

$$
\hat{\nabla}_{X}^{(k)} Y=\nabla_{X} Y+g(\phi A X, Y) \xi-\eta(Y) \phi A X-k \eta(X) \phi Y
$$

for any X,Y tangent to $M$. Then $\hat{\nabla}^{(k)} \eta=0, \hat{\nabla}^{(k)} \xi=0, \hat{\nabla}^{(k)} g=0, \hat{\nabla}^{(k)} \phi=0$. In particular, if the shape operator of a real hypersurface satisfies $\phi A+A \phi=2 k \phi, M$ is a contact manifold and the $k$-th generalized Tanaka-Webster connection coincides with the Tanaka-Webster connection. From (1.1) the $k$-th Cho operator on $M$ associated to a tangent vector field $X$ is defined by $F_{X}^{(k)} Y=g(\phi A X, Y) \xi-\eta(Y) \phi A X-k \eta(X) \phi Y$, for any $Y$ tangent to $M$. The torsion of the $k$-th generalized Tanaka-Webster connection is then given by $T^{(k)}(X, Y)=F_{X}^{(k)} Y-F_{Y}^{(k)} X$. For any $X$ tangent to $M$ we call $T_{X}^{(k)}$ to the operator on $M$ given by $T_{X}^{(k)} Y=T^{(k)}(X, Y)$, for any $Y$ tangent to $M$ and call it the torsion operator associated to $X$.

Jacobi fields along geodesics of a given Riemannian manifold $(\tilde{M}, \tilde{g})$ satisfy a very well-known differential equation. This classical differential equation naturally inspires the so-called Jacobi operator. That is, if $\tilde{R}$ is the curvature operator of $\tilde{M}$, and $X$ is any tangent vector field to $\tilde{M}$, the Jacobi operator (with respect to $X$ ) at $p \in M$, $\tilde{R}_{X} \in \operatorname{End}\left(T_{p} \tilde{M}\right)$, is defined as $\left(\tilde{R}_{X} Y\right)(p)=(\tilde{R}(Y, X) X)(p)$ for all $Y \in T_{p} \tilde{M}$, being a selfadjoint endomorphism of the tangent bundle $T \tilde{M}$ of $\tilde{M}$. Clearly, each tangent vector field $X$ to $\tilde{M}$ provides a Jacobi operator with respect to $X$.

If $M$ is a real hypersurface of $\mathbb{C} P^{m}$ the Jacobi operator associated to the structure 
vector field $\xi$ is called the structure Jacobi operator on $M$ and we denote it by $R_{\xi}$.

Let $\mathcal{L}$ denote the Lie derivative on $M$. Therefore $\mathcal{L}_{X} Y=\nabla_{X} Y-\nabla_{Y} X$ for any $X, Y$ tangent to $M$. If we consider $\hat{\nabla}^{(k)}$, we can define a differential operator of first order $\mathcal{L}^{(k)}$ on $M$ by $\mathcal{L}_{X}^{(k)} Y=\hat{\nabla}_{X}^{(k)} Y-\hat{\nabla}_{Y}^{(k)} X=\mathcal{L}_{X} Y+T_{X}^{(k)} Y$, for any $X, Y$ tangent to $M$.

We can associate to $R_{\xi}$ a tensor field of type $(1,2), R_{\xi_{T}}^{(k)}$, given by $R_{\xi_{T}}^{(k)}(X, Y)=$ $\left[T_{X}^{(k)}, R_{\xi}\right] Y=T_{X}^{(k)} R_{\xi} Y-R_{\xi} T_{X}^{(k)} Y$, for any $X, Y$ tangent to $M$.

In the first paper of this series, [9], first author studied the condition $\mathcal{L}^{(k)} R_{\xi}=$ $\mathcal{L} R_{\xi}$, that is, $\left(\mathcal{L}_{X}^{(k)} R_{\xi}\right) Y=\left(\mathcal{L}_{X} R_{\xi}\right) Y$ for any $X, Y$ tangent to $M$. This condition is equivalent to the fact that $R_{\xi_{T}}^{(k)}$ vanishes, obtaining the following

Theorem There do not exist real hypersurfaces in $\mathbb{C} P^{m}, m \geq 3$, such that $\mathcal{L}^{(k)} R_{\xi}=$ $\mathcal{L} R_{\xi}$.

The purpose of this paper is to study weaker conditions on $M$. Indeed, we will consider real hypersurfaces $M$ in $\mathbb{C} P^{m}$ whose structure Jacobi operator satisfies the following Codazzi condition

$$
\left(\left(\mathcal{L}_{X}^{(k)}-\mathcal{L}_{X}\right) R_{\xi}\right) Y=\left(\left(\mathcal{L}_{Y}^{(k)}-\mathcal{L}_{Y}\right) R_{\xi}\right) X
$$

for any $X, Y$ tangent to $M$. It is easy to see that this condition is equivalent to $R_{\xi_{T}}^{(k)}$ being symmetric, that is, $R_{\xi_{T}}^{(k)}(X, Y)=R_{\xi_{T}}^{(k)}(Y, X)$ for any $X, Y$ tangent to $M$. We will prove the following theorems.

Theorem 1 There does not exist any Hopf real hypersurface $M$ in $\mathbb{C} P^{m}, m \geq 3$, such that $R_{\xi}$ satisfies (1.2) for some nonnull real number $k$.

and

Theorem 2 There does not exist any non-Hopf real hypersurface $M$ in $\mathbb{C} P^{m}, m \geq 3$, such that $R_{\xi}$ satisfies (1.2) for any nonnull real number $k$ such that $k g(A \xi, \xi) \neq-1$.

A different weaker condition is a Killing type condition given by

$$
\left(\left(\mathcal{L}_{X}^{(k)}-\mathcal{L}_{X}\right) R_{\xi}\right) Y+\left(\left(\mathcal{L}_{Y}^{(k)}-\mathcal{L}_{Y}\right) R_{\xi}\right) X=0
$$

for any $X, Y$ tangent to $M$. Now this condition is equivalent to the fact that $R_{\xi_{T}}^{(k)}$ is skew symmetric, that is, $R_{\xi_{T}}^{(k)}(X, Y)=-R_{\xi_{T}}^{(k)}(Y, X)$ for any $X, Y$ tangent to $M$. In this case we have a quite different situation as given by the following results.

Theorem 3 Let $M$ be a Hopf real hypersurface in $\mathbb{C} P^{m}, m \geq 3$, and $k$ a nonnull real number. Then $M$ satisfies (1.3) if and only if either $k=1$ and $M$ is locally congruent to a geodesic hypersphere of radius $\frac{\pi}{4}$ or $k^{2} \geq 4$ and $M$ is locally congruent to a tube 
of radius $r, 0<r<\frac{\pi}{2}$, around $\mathbb{C} P^{n}, 0<n<m-1$, whose radius satisfies either $\cot (r)=k$ or $\cot (r)=-\frac{1}{k}$.

In the non-Hopf case we obtain

Theorem 4 There does not exist any non-Hopf real hypersurface $M$ in $\mathbb{C} P^{m}, m \geq 3$, such that $R_{\xi_{T}}^{(k)}$ is skew symmetric for any nonnull real number $k$ satisfying $\mathrm{kg}(A \xi, \bar{\xi})=$ 1 .

Aknowledgements This work was supported by MINECO-FEDER Project MTM 2016-78807-C2-1-P.

\section{Preliminaries.}

Throughout this paper, all manifolds, vector fields, etc., will be considered of class $C^{\infty}$ unless otherwise stated. Let $M$ be a connected real hypersurface in $\mathbb{C} P^{m}, m \geq 2$, without boundary. Let $N$ be a locally defined unit normal vector field on $M$. Let $\nabla$ be the Levi-Civita connection on $M$ and $(J, g)$ the Kaehlerian structure of $\mathbb{C} P^{m}$.

For any vector field $X$ tangent to $M$ we write $J X=\phi X+\eta(X) N$, where $\phi X$ denotes the tangential component of $J X$ and $-J N=\xi$. Then $(\phi, \xi, \eta, g)$ is an almost contact metric structure on $M$, see [1]. That is, we have

$$
\phi^{2} X=-X+\eta(X) \xi, \quad \eta(\xi)=1, \quad g(\phi X, \phi Y)=g(X, Y)-\eta(X) \eta(Y)
$$

for any tangent vectors $X, Y$ to $M$. From (2.1) we obtain

$$
\phi \xi=0, \quad \eta(X)=g(X, \xi) .
$$

From the parallelism of $J$ we get

$$
\left(\nabla_{X} \phi\right) Y=\eta(Y) A X-g(A X, Y) \xi
$$

and

$$
\nabla_{X} \xi=\phi A X
$$

for any $X, Y$ tangent to $M$, where $A$ denotes the shape operator of the immersion. As the ambient space has holomorphic sectional curvature 4, the equations of Gauss and Codazzi are given, respectively, by 


$$
\begin{array}{r}
R(X, Y) Z=g(Y, Z) X-g(X, Z) Y+g(\phi Y, Z) \phi X-g(\phi X, Z) \phi Y \\
-2 g(\phi X, Y) \phi Z+g(A Y, Z) A X-g(A X, Z) A Y
\end{array}
$$

and

$$
\left(\nabla_{X} A\right) Y-\left(\nabla_{Y} A\right) X=\eta(X) \phi Y-\eta(Y) \phi X-2 g(\phi X, Y) \xi
$$

for any vector fields $X, Y, Z$ tangent to $M$, where $R$ is the curvature tensor of $M$. We will call the maximal holomorphic distribution $\mathbb{D}$ on $M$ to the following one: at any $p \in M, \mathbb{D}(p)=\left\{X \in T_{p} M \mid g(X, \xi)=0\right\}$. If $U \in \mathbb{D}$ we define $\mathbb{D}_{U}=\{X \in \mathbb{D} \mid g(X, U)=$ $g(X, \phi U)=0\}$. We will say that $M$ is Hopf if $\xi$ is principal, that is, $A \xi=\alpha \xi$ for a certain function $\alpha$ on $M$.

From Gauss equation the structure Jacobi operator on $M$ is given by

$$
R_{\xi} X=X-\eta(X) \xi+g(A \xi, \xi) A X-g(A \xi, X) A \xi
$$

for any $X$ tangent to $M$

In the sequel we need the following results:

Theorem 2.1. [7] Let $M$ be a Hopf real hypersurface of $\mathbb{C} P^{m}, m \geq 2$, and let $X \in \mathbb{D}$ such that $A X=\lambda X$. Then $\alpha=g(A \xi, \xi)$ is constant, $2 \lambda-\alpha \neq 0$ and $\phi X$ is principal with principal curvature $\frac{\alpha \lambda+2}{2 \lambda-\alpha}$.

Theorem 2.2. [8] Let $M$ be a real hypersurface of $\mathbb{C} P^{m}, m \geq 2$. Then the following are equivalent:

1. $M$ is locally congruent to a real hypersurface of type $(A)$.

2. $\phi A=A \phi$.

Theorem 2.3 [10] There do not exist real hypersurfaces $M$ in $\mathbb{C} P^{m}, m \geq 3$, whose shape operator is given by $A \xi=\alpha \xi+\beta U, A U=\beta \xi+\frac{\beta^{2}-1}{\alpha} U, A \phi U=-\frac{1}{\alpha} \phi U$, where $U$ is a unit vector field in $\mathbb{D}, \alpha$ and $\beta$ are nonvanishing functions on $M$, the eigenvalues of $A$ in $\mathbb{D}_{U}$ are different from $0,-\frac{1}{\alpha}$ and $\frac{\beta^{2}-1}{\alpha}$ and if $Z \in \mathbb{D}_{U}$ satisfies $A Z=\lambda Z$, then $A \phi Z=\lambda \phi Z$.

Theorem 2.4. [10] There do not exist real hypersurfaces $M$ in $\mathbb{C} P^{m}, m \geq 3$, whose shape operator is given by $A \xi=\xi+\beta U, A U=\beta \xi+\left(\beta^{2}-1\right) U, A \phi U=-\phi U$, for a unit $U \in \mathbb{D}$, being $\beta$ a nonvanishing function, and there exists $Z \in \mathbb{D}_{U}$ such that $A Z=-Z, A \phi Z=-\phi Z$. 


\section{Proofs of Theorems 1 and 2.}

Let $M$ be a real hypersurface in $\mathbb{C} P^{m}$ satisfying (1.2). Then $F_{X}^{(k)} R_{\xi} Y-F_{R_{\xi} Y}^{(k)} X-$ $R_{\xi} F_{X}^{(k)} Y+R_{\xi} F_{Y}^{(k)} X=F_{Y}^{(k)} R_{\xi} X-F_{R_{\xi} X}^{(k)} Y-R_{\xi} F_{Y}^{(k)} X+R_{\xi} F_{X}^{(k)} Y$ for any $X, Y$ tangent to $M$. This yields

$$
\begin{gathered}
g\left(\phi A X, R_{\xi} Y\right) \xi-k \eta(X) \phi R_{\xi} Y-g\left(\phi A R_{\xi} Y, X\right) \xi+\eta(X) \phi A R_{\xi} Y+\eta(Y) R_{\xi} \phi A X \\
+k \eta(X) R_{\xi} \phi Y-\eta(X) R_{\xi} \phi A Y-k \eta(Y) R_{\xi} \phi X=g\left(\phi A Y, R_{\xi} X\right) \xi-k \eta(Y) \phi R_{\xi} X \\
-g\left(\phi A R_{\xi} X, Y\right) \xi+\eta(Y) \phi A R_{\xi} X+\eta(X) R_{\xi} \phi A Y+k \eta(Y) R_{\xi} \phi X-\eta(Y) R_{\xi} \phi A X \\
-k \eta(X) R_{\xi} \phi Y
\end{gathered}
$$

for any $X, Y$ tangent to $M$.

If in (3.1) we take $X, Y \in \mathbb{D}$ we obtain

$$
g\left(\phi A X, R_{\xi} Y\right) \xi-g\left(\phi A R_{\xi} Y, X\right) \xi=g\left(\phi A Y, R_{\xi} X\right) \xi-g\left(\phi A R_{\xi} X, Y\right) \xi
$$

for any $X, Y \in \mathbb{D}$. And if we take $X=\xi, Y \in \mathbb{D}$ in (3.1) it follows

$$
g\left(\phi A \xi, R_{\xi} Y\right) \xi-k \phi R_{\xi} Y+\phi A R_{\xi} Y+2 k R_{\xi} \phi Y-2 R_{\xi} \phi A Y=0
$$

for any $Y \in \mathbb{D}$.

Let us suppose that $M$ is Hopf with $A \xi=\alpha \xi$. Then (3.3) gives

$$
-k \phi R_{\xi} Y+\phi A R_{\xi} Y+2 k R_{\xi} \phi Y-2 R_{\xi} \phi A Y=0
$$

for any $Y \in \mathbb{D}$. If $X \in \mathbb{D}$ then $R_{\xi} X=X+\alpha A X$. Therefore, (3.2) yields $g(\phi A X, Y+$ $\alpha A Y)-g\left(\phi A Y+\alpha \phi A^{2} Y, X\right)=g(\phi A Y, X+\alpha A X)-g\left(\phi A X+\alpha \phi A^{2} X, Y\right.$ for any $X, Y \in$ $\mathbb{D}$. Then $2 g(\phi A X, Y)+2 \alpha g(\phi A X, A Y)-2 g(\phi A Y, X)-\alpha g\left(\phi A^{2} Y, X\right)+\alpha g\left(\phi A^{2} X, Y\right)=$ 0 , for any $X, Y \in \mathbb{D}$. As $M$ is Hopf this implies

$$
2 \phi A X+2 \alpha A \phi A X+2 A \phi X+\alpha A^{2} \phi X+\alpha \phi A^{2} X=0
$$

for any $X \in \mathbb{D}$. Let now $X \in \mathbb{D}$ such that $A X=\lambda X$. Then $A \phi X=\mu \phi X$, $\mu=\frac{\alpha \lambda+2}{2 \lambda-\alpha}$. Then (3.5) yields $2 \lambda+2 \alpha \lambda \mu+2 \mu+\alpha \mu^{2}+\alpha \lambda^{2}=0$. That is, $2(\lambda+$ 
$\mu)+\alpha(\lambda+\mu)^{2}=0$. Thus if we suppose $\lambda+\mu=0, \frac{2 \lambda^{2}+2}{2 \lambda-\alpha}=0$, which is impossible. Therefore,

$$
\alpha \neq 0
$$

and $\alpha(\lambda+\mu)=-2$. That is, $\frac{2 \lambda^{2}+2}{2 \lambda-\alpha}=-\frac{2}{\alpha}$. This implies $\left(\lambda^{2}+1\right) \alpha=\alpha-2 \lambda$, that is, $\lambda^{2} \alpha=-2 \lambda$. Therefore, either $\lambda=0$ or $\lambda=-\frac{2}{\alpha}$. If $X$ satisfies $A X=0$, then $A \phi X=-\frac{2}{\alpha} \phi X$. This implies $R_{\xi} X=X$ and $R_{\xi} \phi X=\phi X+\alpha A \phi X=-\phi X$. From (3.4) we get $-k \phi X-2 k \phi X=0$, that is, $3 k \phi X=0$, which is impossible. We obtain a similar situation if we suppose that $A X=-\frac{2}{\alpha} X$ and Theorem 1 is proved.

Suppose now that $M$ is non-Hopf. Then, at least on a neighbourhood of a certain point of $M$ we can find a unit vector field $U \in \mathbb{D}$ and functions $\alpha$ and $\beta$, where $\beta$ does not vanish, such that we can write $A \xi=\alpha \xi+\beta U$. From now on we consider all the calculations on such a neighborhood. Then (3.3) gives

$$
\beta g\left(\phi U, R_{\xi} Y\right) \xi-k \phi R_{\xi} Y+\phi A R_{\xi} Y+2 k R_{\xi} \phi Y-2 R_{\xi} \phi A Y=0
$$

for any $Y \in \mathbb{D}$. Its scalar product with $\xi$ yields $\beta g\left(R_{\xi} \phi U, Y\right)=0$ for any $Y \in \mathbb{D}$. As $R_{\xi} \xi=0$ we obtain $R_{\xi} \phi U=0$ and then

$$
A \phi U=-\frac{1}{\alpha} \phi U
$$

If in (3.7) we take $Y=\phi U$ we get $-2 k R_{\xi} U+\frac{2}{\alpha} R_{\xi} \phi^{2} U=0$. Thus $-\left(2 k+\frac{2}{\alpha}\right) R_{\xi} U=0$ and, as we suppose that $\alpha \neq-\frac{1}{k}$ we obtain $R_{\xi} U=0$ or

$$
A U=\beta \xi+\frac{\beta^{2}-1}{\alpha} U .
$$

Therefore, $\mathbb{D}_{U}$ is $A$-invariant. From (3.2) we know that $g\left((\phi A+A \phi) X, R_{\xi} Y\right)=$ $g\left((\phi A+A \phi) Y, R_{\xi} X\right)$ for any $X, Y \in \mathbb{D}_{U}$. Therefore,

$$
-(\phi A+A \phi) R_{\xi} Y=R_{\xi}(\phi A+A \phi) Y
$$


for any $Y \in \mathbb{D}_{U}$. If we take $Y \in \mathbb{D}_{U}$ unit and such that $A Y=\lambda Y$ in (3.7) we have $-k \phi R_{\xi} Y+\phi A R_{\xi} Y+2(k-\lambda) R_{\xi} \phi Y=0$. Then, $-(1+\alpha \lambda) k \phi Y+(1+\alpha \lambda) \phi A Y+2(k-$ $\lambda)(\phi Y+\alpha A \phi Y)=0$. That is, $((1+\alpha \lambda)(\lambda-k)+2(k-\lambda)) \phi Y+2 \alpha(k-\lambda) A \phi Y=0$. Thus $2 \alpha(k-\lambda) A \phi Y=(\alpha \lambda-1)(k-\lambda) \phi Y$. Therefore, either $k=\lambda$ or if $\lambda \neq k$,

$$
A \phi Y=\frac{\alpha \lambda-1}{2 \alpha} \phi Y
$$

We will call $\mu=\frac{\alpha \lambda-1}{2 \alpha}$. If in (3.7) we take $\phi Y$ instead of $Y$ we have $-k \phi R_{\xi} \phi Y+$ $\phi A R_{\xi} \phi Y-2 k R_{\xi} Y-2 R_{\xi} \phi A \phi Y=0$. This implies $-k(1+\alpha \mu) \phi^{2} Y+(1+\alpha \mu) \phi A \phi Y-$ $2 k(1+\alpha \lambda) Y+2 \mu(1+\alpha \lambda) Y=0$. Then $(-1+\alpha \mu-2 \alpha \lambda)(k-\mu)=0$. This gives, bearing in mind the value of $\mu,-3(1+\alpha \lambda)(k-\mu)=0$. Therefore, we have two possibilities: either $\lambda=-\frac{1}{\alpha}$ and then $\mu=-\frac{1}{\alpha}$, or $\mu=k$ and, as we suppose $\lambda \neq k$, in this case $\alpha \lambda-1=2 k \alpha$ and $\lambda=\frac{2 \alpha k+1}{\alpha}$.

As $-(\phi A+A \phi) R_{\xi} Y=R_{\xi}(\phi A+A \phi) Y$, we have $-(1+\alpha \lambda)(\phi A+A \phi) Y=(\lambda+$ $\mu) R_{\xi} \phi Y$. Then $-(1+\alpha \lambda)(\lambda+\mu)=(1+\alpha \mu)(\lambda+\mu)$. If $\lambda=\mu=-\frac{1}{\alpha}$, we obtain $\lambda+\mu \neq 0$.

If $\mu=k$ and we suppose $\lambda+\mu \neq 0$, it follows $-1-\alpha \lambda=1+\alpha \mu$ and $\lambda+\mu=-\frac{2}{\alpha}$. Then $\frac{2 \alpha k+1}{\alpha}+k=\frac{3 \alpha k+1}{\alpha}=-\frac{2}{\alpha}$ yields $3 \alpha k=-3$ and this contradicts the fact of $\alpha k \neq-1$. Therefore, $\lambda+\mu=0$ and $3 \alpha k+1=0$ yields $\alpha=-\frac{1}{3 k}$. In this case, $\alpha, \lambda$ and $\mu$ are constant.

If the unique principal curvature on $\mathbb{D}_{U}$ is $k$, as $-(1+\alpha k)(\phi A+A \phi) Y=2 k(1+$ $\alpha k) \phi Y$, we should have $4 k(1+\alpha k)=0$ and, as $k \neq 0, \alpha k=-1$, which is impossible. Therefore, on $\mathbb{D}_{U}$ there must be, at least, a principal curvature $\lambda$ different from $k$.

Let us suppose that on $\mathbb{D}_{U}$ there is a principal curvature $\lambda=-\frac{1}{\alpha}$ and let $Y$ be a unit eigenvector corresponding to $\lambda$. As we have seen $A \phi Y=-\frac{1}{\alpha} \phi Y$ and the equation of Codazzi implies $\left(\nabla_{Y} A\right) \phi Y-\left(\nabla_{\phi Y} A\right) Y=-2 \xi$. This gives $-Y\left(\frac{1}{\alpha}\right) \phi Y-\frac{1}{\alpha} \nabla_{Y} \phi Y-$ $A \nabla_{Y} \phi Y+(\phi Y)\left(\frac{1}{\alpha}\right) Y+\frac{1}{\alpha} \nabla_{\phi Y} Y+A \nabla_{\phi Y} Y=-2 \xi$. If we take its scalar product with $\xi$ we obtain $\frac{1}{\alpha} g(\phi Y, \phi A Y)-g\left(\nabla_{Y} \phi Y, \alpha \xi+\beta U\right)-\frac{1}{\alpha} g(Y, \phi A \phi Y)+g\left(\nabla_{\phi Y} Y, \alpha \xi+\beta U\right)=-2$. This implies

$$
\beta g([\phi Y, Y], U)=\frac{2}{\alpha^{2}}
$$


And its scalar product with $U$ yields $-\frac{1}{\alpha} g\left(\nabla_{Y} \phi Y, U\right)+\frac{1}{\alpha} g\left(\nabla_{\phi Y} Y, U\right)-g\left(\nabla_{Y} \phi Y, \beta \xi+\right.$ $\left.\frac{\beta^{2}-1}{\alpha} U\right)+g\left(\nabla_{\phi Y} Y, \beta \xi+\frac{\beta^{2}-1}{\alpha} U\right)=0$. This gives $\frac{\beta^{2}}{\alpha} g([\phi Y, Y], U)-\frac{2 \beta}{\alpha}=0$. Thus

$$
\beta g([\phi Y, Y], U)=2 .
$$

Fron (3.12) and (3.13) we have $\alpha^{2}=1$ and, taking $-\xi$ instead of $\xi$ if necessary, we can suppose $\alpha=1$. From Theorem 2.4 this kind of real hypersurfaces does not exist.

Now the unique principal curvatures on $\mathbb{D}_{U}$ are $k$ and $-k$, with $A \xi=-\frac{1}{3 k} \xi+\beta U$, $A U=\beta \xi+3 k\left(1-\beta^{2}\right) U, A \phi U=3 k \phi U$. Let a unit $X \in \mathbb{D}_{U}$ such that $A X=\lambda X$, where we know that $\lambda$ is constant. Then we have $\left(\nabla_{X} A\right) \xi-\left(\nabla_{\xi} A\right) X=-\phi X$, that is, $\nabla_{X}\left(-\frac{1}{3 k} \xi+\beta U\right)-A \phi A X-\nabla_{\xi}(\lambda X)+A \nabla_{\xi} X=-\phi X$. Its scalar product with $\xi$ implies $g\left(\nabla_{\xi} X,-\frac{1}{3 k} \xi+\beta U\right)=0$. As we suppose $\beta \neq 0$, we obtain $g\left(\nabla_{\xi} X, U\right)=0$. But its scalar product with $U$ gives $X(\beta)-\lambda g\left(\nabla_{\xi} X, U\right)+g\left(\nabla_{\xi} X, \beta \xi+3 k\left(\beta^{2}-1\right) U\right)=0$. Then, $X(\beta)+\left(3 k\left(1-\beta^{2}\right)-\lambda\right) g\left(\nabla_{\xi} X, U\right)=0$. this yields

$$
X(\beta)=0
$$

for any $X \in \mathbb{D}_{U}$.

On the other hand $\left(\nabla_{U} A\right) \xi-\left(\nabla_{\xi} A\right) U=-\phi U$ gives $-\frac{1}{3 k} \phi A U+U(\beta) U+\beta \nabla_{U} U-$ $A \phi A U-\xi(\beta) \xi-\beta \phi A \xi-3 k \xi\left(1-\beta^{2}\right) U-3 k\left(1-\beta^{2}\right) \nabla_{\xi} U+A \nabla_{\xi} U=\phi U$. Its scalar product with $\xi$ implies $-\xi(\beta)+g\left(\nabla_{\xi} U,-\frac{1}{3 k} \xi+\beta U\right)=0$. Therefore,

$$
\xi(\beta)=0
$$

From the scalar product of the above expression and $U$ we get $U(\beta)-3 k \xi\left(1-\beta^{2}\right)+$ $g\left(\nabla_{\xi} U, \beta \xi+3 k\left(1-\beta^{2}\right) U\right)=0$. From (3.15) we have

$$
U(\beta)=0
$$

Once more, Codazzi equation implies $\left(\nabla_{\phi U} A\right) \xi-\left(\nabla_{\xi} A\right) \phi U=U$, that is, $-\frac{1}{3 k} \phi A \phi U+$ $(\phi U)(\beta) U+\beta \nabla_{\phi U} U+3 k A U-3 k \nabla_{\xi} \phi U+A \nabla_{\xi} \phi U=U$. Its scalar product with $\xi$ gives $-\beta g(U, \phi A \phi U)+3 k \beta+3 k g(\phi U, \phi A \xi)+g\left(\nabla_{\xi} \phi U,-\frac{1}{3 k} \xi+\beta U\right)=0$. Thus $9 k \beta+\frac{1}{3 k} g(\phi U, \phi A \xi)+\beta g\left(\nabla_{\xi} \phi U, U\right)=0$. As $\beta \neq 0$ we obtain 


$$
g\left(\nabla_{\xi} U, \phi U\right)=9 k+\frac{1}{3 k}
$$

Its scalar product with $U$ yields $\frac{1}{3 k} g(A \phi U, \phi U)+(\phi U)(\beta)+9 k^{2}\left(1-\beta^{2}\right)-3 k g\left(\nabla_{\xi} \phi U, U\right)+$ $g\left(\nabla_{\xi} \phi U, \beta \xi+3 k\left(1-\beta^{2}\right) U\right)=1$. From (3.17) we obtain $(\phi U)(\beta)+9 k^{2}-9 k^{2} \beta^{2}+27 k^{2}+$ $1-\beta^{2}+3 k\left(\beta^{2}-1\right)\left(9 k+\frac{1}{3 k}\right)=0$ and this implies

$$
(\phi U)(\beta)=-9 k^{2}\left(1+2 \beta^{2}\right) .
$$

From (3.14), (3.15), (3.16) and (3.18) we obtain

$$
\operatorname{grad}(\beta)=\omega \phi U
$$

where $\omega=-9 k^{2}\left(1+2 \beta^{2}\right)$.

As $g\left(\nabla_{X} \operatorname{grad}(\beta), Y\right)=g\left(\nabla_{Y} \operatorname{grad}(\beta), X\right)$, for any $X, Y$ tangent to $M$, we obtain $X(\omega) g(\phi U, Y)+\omega g\left(\nabla_{X} \phi U, Y\right)=Y(\omega) g(\phi U, X)+\omega g\left(\nabla_{Y} \phi U, X\right)$, for any $X, Y$ tangent to $M$. Taking $Y=\xi$ we get $\omega g\left(\nabla_{X} \phi U, \xi\right)=\omega g\left(\nabla_{\xi} \phi U, X\right)$, that is, $-\omega g(U, A X)=$ $\omega g\left(\nabla_{\xi} \phi U, X\right)$ for any $X$ tangent to $M$. If $X=U$ we have $-\omega g(A U, U)=\omega g\left(\nabla_{\xi} \phi U, U\right)=$ $-\omega g\left(\nabla_{\xi} U, \phi U\right)$. Therefore, either $\omega=0$, which is impossible, or $g(A U, U)=g\left(\nabla_{\xi} U, \phi U\right)$. This yields $3 k\left(1-\beta^{2}\right)=9 k+\frac{1}{3 k}$, that is, $1-\beta^{2}=3+\frac{1}{9 k^{2}}$. Equivalently, $\beta^{2}=-2-\frac{1}{9 k^{2}}$, which is impossible. This finishes the proof of Theorem 2.

\section{Proofs of Theorems 3 and 4.}

If $M$ is a real hypersurface satisfying (1.3) we obtain

$$
\begin{gathered}
g\left(\phi A X, R_{\xi} Y\right) \xi-k \eta(X) \phi R_{\xi} Y-g\left(\phi A R_{\xi} Y, X\right) \xi+\eta(X) \phi A R_{\xi} Y \\
+\eta(Y) R_{\xi} \phi A X+k \eta(X) R_{\xi} \phi Y-\eta(X) R_{\xi} \phi A Y-k \eta(Y) R_{\xi} \phi X+g\left(\phi A Y, R_{\xi} X\right) \xi \\
-k \eta(Y) \phi R_{\xi} X-g\left(\phi A R_{\xi} X, Y\right) \xi+\eta(Y) \phi A R_{\xi} X+\eta(X) R_{\xi} \phi A Y \\
+k \eta(Y) R_{\xi} \phi X-\eta(Y) R_{\xi} \phi A X-k \eta(X) R_{\xi} \phi Y=0
\end{gathered}
$$

for any $X, Y$ tangent to $M$. If in (4.1) we take $X, Y \in \mathbb{D}$ we have

$$
g\left(\phi A X, R_{\xi} Y\right) \xi-g\left(\phi A R_{\xi} Y, X\right) \xi+g\left(\phi A Y, R_{\xi} X\right) \xi-g\left(\phi A R_{\xi} X, Y\right) \xi=0
$$


for any $X, Y \in \mathbb{D}$. Taking $X=\xi, Y \in \mathbb{D}$ in (4.1) we get $g\left(\phi A \xi, R_{\xi} Y\right) \xi-k \phi R_{\xi} Y+$ $\phi A R_{\xi} Y+k R_{\xi} \phi Y-R_{\xi} \phi A Y+R_{\xi} \phi A Y-k R_{\xi} \phi Y=0$. This gives

$$
g\left(\phi A \xi, R_{\xi} Y\right) \xi-k \phi R_{\xi} Y+\phi A R_{\xi} Y=0
$$

for any $Y \in \mathbb{D}$.

Let us suppose that $M$ is Hopf and $A \xi=\alpha \xi$. From (4.2) we get $-A \phi R_{\xi} Y-$ $\phi A R_{\xi} Y+R_{\xi} \phi A Y+R_{\xi} A \phi Y=0$ for any $Y \in \mathbb{D}$. Take a unit $Y \in \mathbb{D}$ such that $A Y=\lambda Y$. Then $A \phi Y=\mu \phi Y, \mu=\frac{\alpha \lambda+2}{2 \lambda-\alpha}$. In this case, $R_{\xi} Y=(1+\alpha \lambda) Y$ and $R_{\xi} \phi Y=(1+\alpha \mu) \phi Y$. From (4.3) we have $-k(1+\alpha \lambda) \phi Y+\lambda(1+\alpha \lambda) \phi Y=0$. Thus $(\lambda-k)(1+\alpha \lambda)=0$. Therefore, if $\alpha=0, \lambda=k$ and if $\alpha \neq 0$, either $\lambda=k$ or $\lambda=-\frac{1}{\alpha}$. But as $-A \phi R_{\xi} Y-\phi A R_{\xi} Y+R_{\xi} \phi A Y+R_{\xi} A \phi Y=0$ we get $-(1+\alpha \lambda) \mu \phi Y-$ $(1+\alpha \lambda) \lambda \phi Y-\lambda(1+\alpha \mu) \phi Y+\mu(1+\alpha \mu) \phi Y=0$. That is, $(\lambda+\mu)(\mu-\lambda) \alpha=0$. As $\lambda+\mu=\lambda+\frac{\alpha \lambda+2}{2 \lambda-\alpha}=\frac{2 \lambda^{2}+2}{2 \lambda-\alpha} \neq 0$, it follows $\alpha(\mu-\lambda)=0$.

If $\alpha=0$ the unique principal curvature on $\mathbb{D}$ is $k$. From [3] $M$ must be locally congruent to a geodesic hypersphere of radius $\frac{\pi}{4}$. Therefore $k=\cot \left(\frac{\pi}{4}\right)=1$.

If $\alpha \neq 0, \lambda=\mu$, that is $\phi A=A \phi$ and from Theorem 2.2, $M$ must be locally congruent to a real hypersurface of type $(A)$. Its principal curvatures are $\alpha,-\frac{1}{\alpha}$ and $k$. In the case of a geodesic hypersphere we should have $\alpha=-\frac{1}{k}$. Thus $2 \cot (2 r)=-\frac{1}{k}$ and $\cot (r)=k$. Thus $-\frac{1}{k}=2 \cot (2 r)=\cot (r)-\tan (r)=k-\frac{1}{k}$ yields $k=0$, which is impossible.

Therefore, $M$ is locally congruent to a tube of radius $r, 0<r<\frac{\pi}{4}$, around $\mathbb{C} P^{n}$, $0<n<m-1$. Their principal curvatures are $\alpha=2 \cot (2 r)=\cot (r)-\tan (r)$, $\cot (r)$ and $-\tan (r)$. We thus have two situations: either $\cot (r)=k$ and $\tan (r)=\frac{1}{\alpha}$, $\alpha=k-\frac{1}{\alpha}$, or $\cot (r)=-\frac{1}{\alpha}, \tan (r)=-k$ and again $\alpha=-\frac{1}{\alpha}+k$. Anyway, $\alpha^{2}-k \alpha+1=0$, Thus $\alpha=\frac{k \pm \sqrt{k^{2}-4}}{2}$. Therefore, $k^{2} \geq 4$ and either $\cot (r)=k$ or $\cot (r)=-\frac{1}{k}$, finishing the proof of Theorem 3 .

Let us suppose now that $M$ is non-Hopf and write, as in previous section, $A \xi=$ $\alpha \xi+\beta U$. Then (4.3) gives

$$
\beta g\left(\phi U, R_{\xi} Y\right) \xi-k \phi R_{\xi} Y+\phi A R_{\xi} Y=0
$$


for any $Y \in \mathbb{D}$. The scalar product of (4.4) and $\xi$ yields $\beta g\left(R_{\xi} \phi U, Y\right)=0$ for any $Y \in \mathbb{D}$ and, being $\beta \neq 0$ and $R_{\xi} \xi=0$, we obtain $R_{\xi} \phi U=0$, that is, $\phi U+\alpha A \phi U=0$. Then

$$
\alpha \neq 0
$$

and

$$
A \phi U=-\frac{1}{\alpha} \phi U
$$

Also (4.4) becomes

$$
\phi A R_{\xi} Y=k \phi R_{\xi} Y
$$

for any $Y \in \mathbb{D}$. If we take (4.7) into (4.2) we obtain $g\left(\phi A X, R_{\xi} Y\right)-k g\left(\phi R_{\xi} Y, X\right)+$ $g\left(\phi A Y, R_{\xi} X\right)-k g\left(\phi R_{\xi} X, Y\right)=0$ for any $X, Y \in \mathbb{D}$. As $g\left(R_{\xi} \phi A X+k R_{\xi} \phi X-A \phi R_{\xi} X-\right.$ $\left.k \phi R_{\xi} X, \xi\right)=0$ because $R_{\xi} \phi U=0$, we get

$$
R_{\xi} \phi A X+k R_{\xi} \phi X-A \phi R_{\xi} X-k \phi R_{\xi} X=0
$$

for any $X \in \mathbb{D}$. Taking $X=\phi U$ in (4.8) we have $R_{\xi} \phi A \phi U-k R_{\xi} U=0$. From (4.6) it follows $\left(\frac{1}{\alpha}-k\right) R_{\xi} U=0$. As we suppose $k \alpha \neq 1$ we obtain $R_{\xi} U=0$ and then

$$
A U=\beta \xi+\frac{\beta^{2}-1}{\alpha} U .
$$

Now $\mathbb{D}_{U}$ is A-invariant. Take a unit $Y \in \mathbb{D}_{U}$ such that $A Y=\lambda Y$. Then $R_{\xi} Y=(1+$ $\alpha \lambda) Y$ and applying $\phi$ to (4.7) we have $-A R_{\xi} Y+g\left(A R_{\xi} Y, \xi\right) \xi=-k R_{\xi} Y+k g\left(R_{\xi} Y, \xi\right) \xi$. As $R_{\xi} U=0$ this yields $A R_{\xi} Y=k R_{\xi} Y$. Therefore $\lambda(1+\alpha \lambda) Y=k(1+\alpha \lambda) Y$. Thus either $\lambda=-\frac{1}{\alpha}$ or $\lambda=k$.

Moreover, from (4.8), for such a $Y$ we get $\lambda R_{\xi} \phi Y+k R_{\xi} \phi Y-(1+\alpha \lambda) A \phi Y-(1+$ $\alpha \lambda) k \phi Y=0$. Now if $\lambda=-\frac{1}{\alpha}$ we arrive to $\left(k-\frac{1}{\alpha}\right) R_{\xi} \phi Y=0$ and, being $k \alpha \neq 1$, we obtain $R_{\xi} \phi Y=0=\phi Y+\alpha A \phi Y$, which implies $A \phi Y=-\frac{1}{\alpha} \phi Y$.

If $\lambda=k$, from (4.8) we have $2 k R_{\xi} \phi Y-(1+k \alpha) A \phi Y-k(1+k \alpha) \phi Y=0$, that is, $2 k(\phi Y+\alpha A \phi Y)-(1+k \alpha) A \phi Y-k(1+k \alpha) \phi Y=0$. Thus $(k \alpha-1) A \phi Y=k(k \alpha-1) \phi Y$ 
and, as $k \alpha \neq 1$ we obtain $A \phi Y=k \phi Y$. We have obtained that any eigenspace in $\mathbb{D}_{U}$ is $\phi$-invariant.

From Theorem 2.4 the case of $\lambda=-\frac{1}{\alpha}$ does not occur and then the unique principal curvature on $\mathbb{D}_{U}$ is $k$. Then, for any $X \in \mathbb{D}_{U}$, Codazzi equation gives $\left(\nabla_{X} A\right) \phi X-$ $\left(\nabla_{\phi X} A\right) X=-2 \xi$. This yields $k \nabla_{X} \phi X-A \nabla_{X} \phi X-k \nabla_{\phi X} X+A \nabla_{\phi X} X=-2 \xi$. Its scalar product with $\xi$ implies $-k g(\phi X, \phi A X)-g\left(\nabla_{X} \phi X, \alpha \xi+\beta U\right)+k g(X, \phi A \phi X)+$ $g\left(\nabla_{\phi X} X, \alpha \xi+\beta U\right)=-2$, that is

$$
\beta g([\phi X, X], U)=2 k^{2}-2 k \alpha-2 \text {. }
$$

Its scalar product with $U$ gives $-k g([\phi X, X], U)-g\left(\nabla_{X} \phi X, \beta \xi+\frac{\beta^{2}-1}{\alpha} U\right)+$ $g\left(\nabla_{\phi X} X, \beta \xi+\frac{\beta^{2}-1}{\alpha} U\right)=0$. This yields

$$
\left(\frac{\beta^{2}-1}{\alpha}-k\right) g([\phi X, X], U)=-2 k \beta
$$

If $k=\frac{\beta^{2}-1}{\alpha}, 2 \beta k=0$, which is impossible. Then $k \neq 0$ and $k \neq \frac{\beta^{2}-1}{\alpha}$. Moreover, from (4.10) and (4.11) we obtain $2\left(k^{2}-k \alpha-1\right)\left(\frac{\beta^{2}-1}{\alpha}-k\right)=-2 k \beta^{2}$. That is, $\left(k^{2}-(k \alpha+1)\right)\left(\beta^{2}-(k \alpha+1)\right)=-k \alpha \beta^{2}$. If $k=-\frac{1}{\alpha}$, we should have $k^{2} \beta^{2}=\beta^{2}$ and therefore, $k^{2}=1$ and $\alpha^{2}=1$. This hypersurfaces do not exist from Theorem 2.4.

Thus the unique principal curvature on $\mathbb{D}_{U}$ is $k \neq 0,-\frac{1}{\alpha}, \frac{\beta^{2}-1}{\alpha}$ and such a real hypersurface does not exist from Theore, 2.3. This finishes our proof.

\section{References}

[1] D.E. Blair, Riemannian Geometry of contact and symplectic manifolds, Progress in Mathematics 203 (2002), Birkhauser Boston Inc. Boston.

[2] J.T. Cho, CR-structures on real hypersurfaces of a complex space form, Publ. Math. Debrecen 54 (1999), 473-487.

[3] T.E. Cecil and P.J. Ryan, Focal sets and real hypersurfaces in complex projective space, Trans. A.M.S. 269 (1982), 481-499.

[4] J.T. Cho, Pseudo-Einstein CR-structures on real hypersurfaces in a complex space form, Hokkaido Math. J. 37 (2008), 1-17. 
[5] I. Jeong, E. Pak and Y.J. Suh, Real hypersurfaces in complex two-plane Grassmannians with generalized Tanaka-Webster invariant shape operator, J. Math. Phys. Anal. Geom. 9 (2013), 360-378.

[6] M. Kimura, Real hypersurfaces and complex submanifolds in complex projective space, Trans. A.M.S. 296 (1986), 137-149.

[7] Y. Maeda, On real hypersurfaces of a complex projective space, J. Math. Soc. Japan 28 (1976), 529.540.

[8] M. Okumura, On some real hypersurfaces of a complex projective space, Trans. A.M.S. 212 (1975), 355-364.

[9] J.D. Pérez, Lie derivatives and structure Jacobi operator on real hypersurfaces in complex projective spaces, Diff. Geom. Appl. 50 (2017), 1-10.

[10] J.D. Pérez and F.G. Santos, Real hypersurfaces in complex projective space whose structure Jacobi operator satisfies $\mathcal{L}_{\xi} R_{\xi}=\nabla_{\xi} R_{\xi}$, Rocky Mount. J. Math. 39 (2009), 1293-1301.

[11] R. Takagi, On homogeneous real hypersurfaces in a complex projective space, Osaka J. Math. 10 (1973), 495-506.

[12] R. Takagi, Real hypersurfaces in complex projective space with constant principal curvatures, J. Math. Soc. Japan 27 (1975), 43-53.

[13] R. Takagi, Real hypersurfaces in complex projective space with constant principal curvatures II, J. Math. Soc. Japan 27 (1975), 507-516.

[14] N. Tanaka, On non-degenerate real hypersurfaces, graded Lie algebras and Cartan connections, Japan. J. Math. 2 (1976), 131-190.

[15] S. Tanno, Variational problems on contact Riemennian manifolds, Trans. A.M.S. 314 (1989), 349-379.

[16] S.M. Webster, Pseudohermitian structures on a real hypersurface, J. Diff. Geom. 13 (1978), 25-41.

JuAN DE DiOs PEREZ: JDPEREZ@UGR.ES

Departamento de Geometria y Topologia and IEMATH

UNIVERSIDAD DE GRANADA

18071 GRANADA

SPAIN 
DAvid Perez-Lopez: DAVIDPl109@CORReo.UGr.eS

Fundación I+D DEL Software LiBre - FIDESOL

Avda. de la Innovación, 1 - Ed. BIC CEEI (PTS)

18016 Armilla (Granada)

SPAIN 